\title{
ON THE DETERMINANT OF THE SECOND DERIVATIVE OF A LAPLACE TRANSFORM
}

\author{
By CÉlestin C. Kokonendji and V. Seshadri ${ }^{1}$ \\ Université Paul Sabatier and McGill University
}

\begin{abstract}
If $\mu$ is a positive measure on $\mathbb{R}^{n}$ with Laplace transform $L_{\mu}$, we show that there exists a positive measure $\nu$ on $\mathbb{R}^{n}$ such that $\operatorname{det} L_{\mu}^{\prime \prime}=L_{\nu}$. We deduce various corollaries from this result and, in particular, we obtain the Rao-Blackwell estimator of the determinant of the variance of a natural exponential family on $\mathbb{R}^{n}$ based on $(n+1)$ observations. A new proof and extensions of Lindsay's results on the determinants of moment matrices are also given. Finally we give a characterization of the Gaussian law in $\mathbb{R}^{n}$.
\end{abstract}

1. Introduction. If $\mu$ is a finite non-negative measure on $\mathbb{R}$, with moment generating function $m(t)$ (assumed to be finite on an open interval containing zero), Lindsay (1989) has shown that the determinant of the Hankel matrix $M_{p}(t)=\left(m^{(i+j)}(t)\right)$, whose entries are derivatives of $m(t)$, is itself, as a function of $t$, a moment generating function of another finite non-negative measure $\nu$ on $\mathbb{R}$. Motivated by this result we consider a generalization to measures on $\mathbb{R}^{n}$.

Let $\mu$ be a positive measure on $\mathbb{R}^{n}$ with Laplace transform

$$
L_{\mu}(\boldsymbol{\theta})=\int_{\mathbb{R}^{n}} \exp \langle\boldsymbol{\theta}, \mathbf{X}\rangle \mu(d \mathbf{X})
$$

and cumulant transform $k_{\mu}(\boldsymbol{\theta})=\log L_{\mu}(\boldsymbol{\theta})$. We suppose that the set of $\boldsymbol{\theta}$ in $\mathbb{R}^{n}$ such that $L_{\mu}(\boldsymbol{\theta})$ exists has a non-empty interior $\Theta(\mu)$ and we denote by $\overline{\mathscr{M}}\left(\mathbb{R}^{n}\right)$ the set of such $\mu$ and by $\mathscr{M}\left(\mathbb{R}^{n}\right)$ the set of such $\mu$ which furthermore are not concentrated on an affine hyperplane of $\mathbb{R}^{n}$. Among the moment matrices of measures on $\mathbb{R}^{n}$, by far the most important is the determinant of

$$
L_{\mu}^{\prime \prime}(\boldsymbol{\theta})=\left[\frac{\partial^{2} L_{\mu}(\boldsymbol{\theta})}{\partial \theta_{i} \partial \theta_{j}}\right]_{i, j=1, \ldots, n},
$$

the determinant of the matrix of second order moments. Our main result shows that this determinant is again a Laplace transform of a positive measure $\nu$ on $\mathbb{R}^{n}$. On applying this result to a suitable $\mu$, we obtain Lindsay's result as Corollary 2.1 and Theorem 2.2. Finally, we apply this to simple

\footnotetext{
Received May 1994; revised November 1994, October 1995.

${ }^{1}$ Research supported by NSERC Grant 283-34 and NATO collaborative research grant 921347.

AMS 1991 subject classifications. 60E10, 62E10.

Key words and phrases. Characterization, determinant, generalized variance, Laplace trans-
} form, natural exponential family, quadratic variance functions. 
quadratic natural exponential families in $\mathbb{R}^{n}$ developed by Casalis (1992, 1994) as a generalization of the Morris (1982) class. We also apply it to Wishart distributions on symmetric matrices. The next application deals with the Rao-Blackwell estimator of the generalized variance of a natural exponential family in $\mathbb{R}^{n}$ in the special case of $(n+1)$ observations. We conclude with a characterization of the Gaussian laws in $\mathbb{R}^{n}$.

2. Main results. In what follows we use the notation $\mathbf{X} \otimes \mathbf{Y}$ to denote the matrix $\left(x_{i} y_{j}\right)_{i, j=1, \ldots, n}$, where $\mathbf{X}=\left(x_{1}, \ldots, x_{n}\right)^{t}$ and $\mathbf{Y}=\left(y_{1}, \ldots, y_{n}\right)^{t} . \mathrm{A}$ prime stands for the first derivative, two primes for the second derivative, $\operatorname{det} \mathbf{A}$ for the determinant of the matrix $\mathbf{A}$ and $\mu^{* k}$ for the $k$-fold convolution of the measure $\mu \in \overline{\mathscr{M}}\left(\mathbb{R}^{n}\right)$.

Our main result is the following.

Theorem 2.1. Let $\mu \in \overline{\mathscr{M}}\left(\mathbb{R}^{n}\right), \mathbf{x}_{1}, \ldots, \mathbf{x}_{n} \in \mathbb{R}^{n}$ and $\nu$ be the image of

by the map

$$
\frac{1}{n !}\left(\operatorname{det}\left[\mathbf{X}_{1}, \ldots, \mathbf{X}_{n}\right]\right)^{2} \mu\left(d \mathbf{X}_{1}\right) \cdots \mu\left(d \mathbf{X}_{n}\right)
$$

$$
S:\left(\mathbb{R}^{n}\right)^{n} \rightarrow \mathbb{R}^{n}, \quad\left(\mathbf{X}_{1}, \ldots, \mathbf{X}_{n}\right) \mapsto \mathbf{X}_{1}+\cdots+\mathbf{X}_{n} .
$$

Then

$$
\operatorname{det} L_{\mu}^{\prime \prime}(\boldsymbol{\theta})=L_{\nu}(\boldsymbol{\theta})
$$

and

$$
\operatorname{det} L_{\mu}^{\prime \prime}(\boldsymbol{\theta})=\left(L_{\mu}(\boldsymbol{\theta})\right)^{n} \operatorname{det}\left[k_{\mu}^{\prime \prime}(\boldsymbol{\theta})+k_{\mu}^{\prime}(\boldsymbol{\theta}) \otimes k_{\mu}^{\prime}(\boldsymbol{\theta})\right] .
$$

As a corollary we obtain a theorem of Lindsay [(1989, Theorem 3A] on the moment matrices of measures on $\mathbb{R}$.

Corollary 2.1 (Lindsay). Let $\alpha \in \mathscr{M}(\mathbb{R})$ and let $\beta$ be the image measure of $(1 / n !) \prod_{i<j}\left(x_{i}-x_{j}\right)^{2} \alpha\left(d x_{1}\right) \cdots \alpha\left(d x_{n}\right)$ by the map $s: \mathbb{R}^{n} \rightarrow \mathbb{R},\left(x_{1}, \ldots, x_{n}\right) \mapsto$ $x_{1}+\cdots+x_{n}$. Then for every $t \in \Theta(\alpha)$,

$$
\operatorname{det}\left[\left(\frac{d}{d t}\right)^{i+j-2} L_{\alpha}(t)\right]_{i, j=1, \ldots, n}=L_{\beta}(t) .
$$

The most important consequence of the above results is an extension of the so-called Lindsay transform [Kokonendji and Seshadri (1992, 1994) and Kokonendji (1993)] of the first order for measures on $\mathbb{R}^{n}$. Let us denote by

$$
\mathscr{L}_{\mu}(\boldsymbol{\theta})=\left[\begin{array}{cc}
L_{\mu}(\boldsymbol{\theta}) & \left(L_{\mu}^{\prime}(\boldsymbol{\theta})\right)^{t} \\
L_{\mu}^{\prime}(\boldsymbol{\theta}) & L_{\mu}^{\prime \prime}(\boldsymbol{\theta})
\end{array}\right]
$$

the symmetric $(n+1) \times(n+1)$ matrix for $\mu \in \mathscr{M}\left(\mathbb{R}^{n}\right)$. We then have the following theorem. 
Theorem 2.2. Let $\mu \in \mathscr{M}\left(\mathbb{R}^{n}\right)$ and let $\nu_{0}$ be the image measure of

$$
\frac{1}{(n+1) !}\left(\operatorname{det}\left[\begin{array}{cccc}
1 & 1 & & 1 \\
\mathbf{X}_{0} & \mathbf{X}_{1} & \cdots & \mathbf{X}_{n}
\end{array}\right]\right)^{2} \mu\left(d \mathbf{X}_{0}\right) \cdots \mu\left(d \mathbf{X}_{n}\right)
$$

by the map

$$
S_{0}:\left(\mathbb{R}^{n}\right)^{n+1} \rightarrow \mathbb{R}^{n}, \quad\left(\mathbf{X}_{0}, \mathbf{X}_{1}, \ldots, \mathbf{X}_{n}\right) \mapsto \mathbf{X}_{0}+\mathbf{X}_{1}+\cdots+\mathbf{X}_{n} .
$$

Then for each $\boldsymbol{\theta} \in \Theta(\mu)$,

$$
\operatorname{det} \mathscr{L}_{\mu}(\boldsymbol{\theta})=L_{\nu_{0}}(\boldsymbol{\theta})
$$

and

$$
\operatorname{det} \mathscr{L}_{\mu}(\boldsymbol{\theta})=\left(L_{\mu}(\boldsymbol{\theta})\right)^{n+1} \operatorname{det} k_{\mu}^{\prime \prime}(\boldsymbol{\theta}) .
$$

The proofs of the theorems rely on the following proposition concerning the expectation of a determinant. This appears as a problem due to Pólya and Szegö (1972), Vol. I, Part II, Chapter 1, Problem 68, pages 61-62, 247]. It generalizes a result (Theorem $2 \mathrm{~A}$ ) mentioned by Lindsay.

Proposition 2.1. Let $\eta$ be a positive measure on $\mathbb{R}^{n} \times \mathbb{R}^{n}$ such that $M=\int_{\mathbb{R}^{n} \times \mathbb{R}^{n}} \mathbf{X} \otimes \mathbf{Y} \eta(d \mathbf{X}, d \mathbf{Y})$ exists. Then

$$
\begin{gathered}
\operatorname{det} M=\frac{1}{n !} \int_{\left(\mathbb{R}^{n} \times \mathbb{R}^{n}\right)^{n}}\left(\operatorname{det}\left[\mathbf{X}_{1} \cdots \mathbf{X}_{n}\right]\right)\left(\operatorname{det}\left[\mathbf{Y}_{1} \cdots \mathbf{Y}_{n}\right]\right) \eta\left(d \mathbf{X}_{1}, d \mathbf{Y}_{1}\right) \cdots \\
\times \eta\left(d \mathbf{X}_{n}, d \mathbf{Y}_{n}\right) .
\end{gathered}
$$

Proof of Theorem 2.1. Let $\boldsymbol{\theta} \in \Theta(\mu)$. Apply Proposition 2.1 to

$$
\eta(d \mathbf{X}, d \mathbf{Y})=\exp \{\langle\boldsymbol{\theta}, \mathbf{X}\rangle\} \mu(d \mathbf{X}) \delta_{\mathbf{X}}(d \mathbf{Y})
$$

$\left(\delta_{\mathbf{X}}\right.$ being Dirac measure at $\left.\mathbf{X}\right)$. Then we obtain on the one hand from the definition of $L_{\mu}^{\prime \prime}(\boldsymbol{\theta})$,

$$
M=\int_{\mathbb{R}^{n}} \mathbf{X} \otimes \mathbf{X} \exp \{\langle\boldsymbol{\theta}, \mathbf{X}\rangle\} \mu(d \mathbf{X})=L_{\mu}^{\prime \prime}(\boldsymbol{\theta}),
$$

while on the other hand, from the definition of $\nu$,

$$
\begin{aligned}
& \frac{1}{n !} \int_{\left(\mathbb{R}^{n}\right)^{n}}\left(\operatorname{det}\left[\mathbf{X}_{1} \cdots \mathbf{X}_{n}\right]\right)^{2} \exp \left\{\left\langle\boldsymbol{\theta}, \mathbf{X}_{1}\right\rangle+\cdots+\left\langle\boldsymbol{\theta}, \mathbf{X}_{n}\right\rangle\right\} \mu\left(d \mathbf{X}_{1}\right) \cdots \mu\left(d \mathbf{X}_{n}\right) \\
& \quad=L_{\nu}(\boldsymbol{\theta}) .
\end{aligned}
$$

This establishes (i).

Since $L_{\mu}(\boldsymbol{\theta})=\exp \left(k_{\mu}(\boldsymbol{\theta})\right)$ we obtain

Hence

$$
L_{\mu}^{\prime \prime}(\boldsymbol{\theta})=L_{\mu}(\boldsymbol{\theta})\left[k_{\mu}^{\prime \prime}(\boldsymbol{\theta})+k_{\mu}^{\prime}(\boldsymbol{\theta}) \otimes k_{\mu}^{\prime}(\boldsymbol{\theta})\right] .
$$

$$
\operatorname{det} L_{\mu}^{\prime \prime}(\boldsymbol{\theta})=\left(L_{\mu}(\boldsymbol{\theta})\right)^{n} \operatorname{det}\left[k_{\mu}^{\prime \prime}(\boldsymbol{\theta})+k_{\mu}^{\prime}(\boldsymbol{\theta}) \otimes k_{\mu}^{\prime}(\boldsymbol{\theta})\right] .
$$

The proof of Corollary 2.1 relies on the following lemma. 
Lemma 2.1. Let $\left(\Omega_{1}, \mathscr{A}_{1}\right)$ and $\left(\Omega_{2}, \mathscr{A}_{2}\right)$ be two measurable spaces, let $g: \Omega_{1} \rightarrow \Omega_{2}$ and $f: \Omega_{2} \rightarrow[0, \infty)$ be two measurable maps and let $\lambda$ and $\lambda_{1}$ be two measures on $\Omega_{1}$ such that

$$
\lambda_{1}\left(d \omega_{1}\right)=f\left(g\left(\omega_{1}\right)\right) \lambda\left(d \omega_{1}\right) .
$$

Then

$$
g\left(\lambda_{1}\right)\left(d \omega_{2}\right)=f\left(\omega_{2}\right) g(\lambda)\left(d \omega_{2}\right) .
$$

Proof. Let $A_{2}$ be in $\mathscr{A}_{2}$. Then

$$
\begin{aligned}
g\left(\lambda_{1}\right)\left(A_{2}\right) & \stackrel{(1)}{=} \lambda_{1}\left(g^{-1}\left(A_{2}\right)\right) \stackrel{(2)}{=} \int_{g^{-1}\left(A_{2}\right)} f\left(g\left(\omega_{1}\right)\right) \lambda\left(d \omega_{1}\right) \\
& \stackrel{(3)}{=} \int_{\Omega_{1}} I_{A_{2}}\left(g\left(\omega_{1}\right)\right) f\left(g\left(\omega_{1}\right)\right) \lambda\left(d \omega_{1}\right) \\
& \stackrel{(4)}{=} \int_{\Omega_{1}} I_{A_{2}}\left(\omega_{2}\right) f\left(\omega_{2}\right) g(\lambda)\left(d \omega_{2}\right) \\
& \stackrel{(5)}{=} \int_{A_{2}} f\left(\omega_{2}\right) g(\lambda)\left(d \omega_{2}\right) .
\end{aligned}
$$

Here (1) is the definition of $g\left(\lambda_{1}\right)\left(A_{2}\right)$, (2) is the definition of $\lambda_{1}$, (3) is a reformulation, (4) is the transport theorem applied to $I_{A_{2}}\left(\omega_{2}\right) f\left(\omega_{2}\right)$ and, finally, (5) is again a reformulation.

Proof of Corollary 2.1. We first define the map $h: \mathbb{R} \rightarrow \mathbb{R}^{n}, x \mapsto$ $\left(1, x, x^{2}, \ldots, x^{n-1}\right)^{t}$ and apply Lemma 2.1 with $\Omega_{1}=\mathbb{R}^{n}, \Omega_{2}=\left(\mathbb{R}^{n}\right)^{n}$,

$$
\begin{aligned}
& g\left(x_{1}, \ldots, x_{n}\right)=\left(h\left(x_{1}\right), \ldots, h\left(x_{n}\right)\right), \\
& f\left(\mathbf{X}_{1}, \ldots, \mathbf{X}_{n}\right)=\frac{1}{n !}\left(\operatorname{det}\left[\mathbf{x}_{1}, \ldots, \mathbf{X}_{n}\right]\right)^{2}
\end{aligned}
$$

and

$$
\lambda\left(d x_{1}, \ldots, d x_{n}\right)=\alpha\left(d x_{1}\right) \cdots \alpha\left(d x_{n}\right) .
$$

A simple application of the Vandermonde determinant then implies that

$$
\lambda_{1}\left(d x_{1}, \ldots, d x_{n}\right)=\frac{1}{n !} \prod_{i<j}\left(x_{i}-x_{j}\right)^{2} \alpha\left(d x_{1}\right) \cdots \alpha\left(d x_{n}\right) .
$$

Lemma 2.1 now gives

$$
g\left(\lambda_{1}\right)\left(d \mathbf{X}_{1}, \ldots, d \mathbf{X}_{n}\right)=\frac{1}{n !}\left(\operatorname{det}\left[\mathbf{x}_{1}, \ldots, \mathbf{X}_{n}\right]\right)^{2} g(\lambda)\left(d \mathbf{X}_{1}, \ldots, d \mathbf{X}_{n}\right),
$$

but

$$
g(\lambda)\left(d \mathbf{X}_{1}, \ldots, d \mathbf{X}_{n}\right)=h(\alpha)\left(d \mathbf{X}_{1}\right) \cdots h(\alpha)\left(d \mathbf{X}_{n}\right)
$$


With the map $S$ : $\left(\mathbf{X}_{1}, \ldots, \mathbf{X}_{n}\right) \mapsto \mathbf{X}_{1}+\cdots+\mathbf{X}_{n}$ applied to $g\left(\lambda_{1}\right)$ we obtain, using Theorem 2.1, the Laplace transform of $S\left(g\left(\lambda_{1}\right)\right)$, namely, $L_{S\left(g\left(\lambda_{1}\right)\right)}(\boldsymbol{\theta})$ as

$$
\begin{aligned}
& \int_{\left(\mathbb{R}^{n}\right)^{n}} \frac{1}{n !}\left(\operatorname{det}\left[\mathbf{X}_{1}, \ldots, \mathbf{X}_{n}\right]\right)^{2} \exp \left\{\left\langle\boldsymbol{\theta}, \mathbf{X}_{1}+\cdots+\mathbf{X}_{n}\right\rangle\right\} h(\alpha)\left(d \mathbf{X}_{1}\right) \cdots h(\alpha)\left(d \mathbf{X}_{n}\right) \\
& \quad=\operatorname{det} L_{h(\alpha)}^{\prime \prime}(\boldsymbol{\theta})
\end{aligned}
$$

From the definition of $h(\alpha)$ we have

$$
L_{h(\alpha)}(\boldsymbol{\theta})=\int_{\mathbb{R}} \exp \left\{\theta_{1}+\theta_{2} x+\cdots+\theta_{n} x^{n-1}\right\} \alpha(d x) .
$$

Hence

$$
\operatorname{det} L_{h(\alpha)}^{\prime \prime}(\boldsymbol{\theta})=\operatorname{det}\left[\int_{\mathbb{R}} x^{i+j-2} \exp \left\{\theta_{1}+\theta_{2} x+\cdots+\theta_{n} x^{n-1}\right\} \alpha(d x)\right]_{i, j=1, \ldots, n} .
$$

We now take $\left(\theta_{1}, \ldots, \theta_{n}\right)=(0, t, 0, \ldots, 0)$ and obtain

$$
\begin{aligned}
\operatorname{det} L_{h(\alpha)}^{\prime \prime}(0, t, 0, \ldots, 0) & =\operatorname{det}\left[\left(\frac{d}{d t}\right)^{i+j-2} L_{\alpha}(t)\right]_{i, j=1, \ldots, n} \\
& =L_{S\left(g\left(\lambda_{1}\right)\right)}(0, t, \ldots, 0)
\end{aligned}
$$

Finally note that if $p$ is the projection map $\left(y_{1}, \ldots, y_{n}\right) \mapsto y_{2}$, then $s$ as defined in Corollary 2.1 is $s=p \circ S \circ g$. Thus $\beta=s\left(\lambda_{1}\right)=p\left(S\left(g\left(\lambda_{1}\right)\right)\right)$. Since $L_{S\left(g\left(\lambda_{1}\right)\right)}(0, t, 0, \ldots, 0)=L_{p\left(S\left(g\left(\lambda_{1}\right)\right)\right)}(t)$, we have

$$
L_{\beta}(t)=\operatorname{det}\left[\left(\frac{d}{d t}\right)^{i+j-2} L_{\mu}(t)\right]_{i, j=1, \ldots, n} .
$$

Proof of Theorem 2.2. We apply Theorem 2.1 to dimension $n+1$ instead of $n$ with $\alpha\left(d x_{0}, \ldots, d x_{n}\right)=\delta_{1}\left(d x_{0}\right) \mu\left(d x_{1}, \ldots, d x_{n}\right)$. Thus if $\boldsymbol{\theta} \in \Theta(\mu)$ and $\theta_{0} \in \mathbb{R}$, we have $L_{\alpha}\left(\theta_{0}, \boldsymbol{\theta}\right)=\exp \left(\theta_{0}\right) L_{\mu}(\boldsymbol{\theta})$ and

$$
L_{\alpha}^{\prime \prime}\left(\theta_{0}, \boldsymbol{\theta}\right)=\exp \left(\theta_{0}\right)\left[\begin{array}{cc}
L_{\mu}(\boldsymbol{\theta}) & \left(L_{\mu}^{\prime}(\boldsymbol{\theta})\right)^{t} \\
L_{\mu}^{\prime}(\boldsymbol{\theta}) & L_{\mu}^{\prime \prime}(\boldsymbol{\theta})
\end{array}\right] .
$$

Setting $\theta_{0}=0$ we obtain (i). The second equality (ii) is obtained by observing that

$$
\begin{aligned}
& L_{\mu}(\boldsymbol{\theta})=\exp k_{\mu}(\boldsymbol{\theta}) \\
& L_{\mu}^{\prime}(\boldsymbol{\theta})=L_{\mu}(\boldsymbol{\theta}) k_{\mu}^{\prime}(\boldsymbol{\theta}) \\
& L_{\mu}^{\prime \prime}(\boldsymbol{\theta})=L_{\mu}(\boldsymbol{\theta})\left[k_{\mu}^{\prime \prime}(\boldsymbol{\theta})+k_{\mu}^{\prime}(\boldsymbol{\theta}) \otimes k_{\mu}^{\prime}(\boldsymbol{\theta})\right]
\end{aligned}
$$


and hence that

$$
\begin{aligned}
\operatorname{det}\left[\begin{array}{cc}
L_{\mu}(\boldsymbol{\theta}) & \left(L_{\mu}^{\prime}(\boldsymbol{\theta})\right)^{t} \\
L_{\mu}^{\prime}(\boldsymbol{\theta}) & \left(L_{\mu}^{\prime}(\boldsymbol{\theta})\right)
\end{array}\right] & =\left(L_{\mu}(\boldsymbol{\theta})\right)^{n+1} \operatorname{det}\left[\begin{array}{cc}
1 & \left(k_{\mu}(\boldsymbol{\theta})\right)^{t} \\
k_{\mu}^{\prime}(\boldsymbol{\theta}) & k_{\mu}^{\prime \prime}(\boldsymbol{\theta})+k_{\mu}^{\prime}(\boldsymbol{\theta}) \otimes k_{\mu}^{\prime}(\boldsymbol{\theta})
\end{array}\right] \\
& =L_{\mu}^{n+1}(\boldsymbol{\theta}) \operatorname{det} k_{\mu}^{\prime}(\boldsymbol{\theta}) .
\end{aligned}
$$

\section{Applications.}

3.1. Quadratic variances. First we give a brief summary of natural exponential families (NEF) in $\mathbb{R}^{n}$ and some important properties associated with them.

To each $\mu \in \mathscr{M}\left(\mathbb{R}^{n}\right)$ and $\boldsymbol{\theta} \in \Theta(\mu)$, the set of probabilities

$$
F=F(\mu)=\{P(\boldsymbol{\theta}, \mu)(d \mathbf{X}) ; \boldsymbol{\theta} \in \Theta(\mu)\},
$$

where

$$
P(\boldsymbol{\theta}, \mu)(d \mathbf{X})=\exp \left\{\langle\boldsymbol{\theta}, \mathbf{X}\rangle-k_{\mu}(\boldsymbol{\theta})\right\} \mu(d \mathbf{X})
$$

is defined as the natural exponential family generated by $\mu$. The measure $\mu$ is said to be a basis of $F$. It is well known that $k_{\mu}(\theta)$ is strictly convex and real analytic on $\Theta(\mu)$ and that its first derivative $k_{\mu}^{\prime}(\boldsymbol{\theta})$, where

$$
k_{\mu}^{\prime}(\boldsymbol{\theta})=\int_{\mathbb{R}^{n}} \mathbf{X} P(\boldsymbol{\theta}, \mu)(d \mathbf{X})
$$

defines a map from $\Theta(\mu)$ to $M_{F}$. If $F=F(\mu)$, then $M_{F}=k_{\mu}^{\prime}(\Theta(\mu))$ is called the mean domain of $F$. The map $\boldsymbol{\theta} \mapsto k_{\mu}^{\prime}(\boldsymbol{\theta})$ is a bijection between $\Theta(\mu)$ and $M_{F}$, and hence we can consider the inverse of $k_{\mu}^{\prime}(\boldsymbol{\theta})$, namely, $\psi_{\mu}: M_{F} \rightarrow \Theta(\mu)$. For each $\mathbf{m} \in M_{F}$ we let $P(\mathbf{m}, F)=P\left(\psi_{\mu}(\mathbf{m}), \mu\right)$ and the bijective map $M_{F} \Rightarrow F, \mathbf{m} \mapsto P(\mathbf{m}, F)$ defines a parametrization of $F$ by the mean.

Since $P(\mathbf{m}, F)$ is a distribution in $\mathbb{R}^{n}$, its covariance matrix is

$$
V_{F}(\mathbf{m})=\int_{\mathbb{R}^{n}}(\mathbf{X}-\mathbf{m})(\mathbf{X}-\mathbf{m})^{t} P(\mathbf{m}, F)(d \mathbf{X})
$$

for each $\mathbf{m} \in M_{F}$.

The $(n \times n)$ matrix $V_{F}(\mathbf{m})$ defined on $M_{F}$ is called the variance function of $P(\mathbf{m}, F)$ or $F$. Note that $V_{F}(\mathbf{m})=k_{\mu}^{\prime \prime}\left(\psi_{\mu}(\mathbf{m})\right)$. In fact, $k_{\mu}^{\prime \prime}(\boldsymbol{\theta})$ is a Hessian operator and in the canonical basis has the matrix representation

$$
\left(\frac{\partial^{2} k_{\mu}}{\partial \theta_{i} \partial \theta_{j}}\right)_{i, j=1, \ldots, n} .
$$

The generalized variance [Wilks (1932)] of $F$ is $\operatorname{det} k_{\mu}^{\prime \prime}(\boldsymbol{\theta})$.

Casalis (1994) introduced natural exponential family (NEF) with variance functions of the form

$$
V_{F}(\mathbf{m})=a \mathbf{m} \otimes \mathbf{m}+B(\mathbf{m})+c,
$$


where $a \in \mathbb{R}, B(\mathbf{m})$ is a matrix $(n \times n)$ of elements linear in $\mathbf{m}$ and $c$ is a matrix $(n \times n)$ of constants. Casalis calls these NEF simple quadratic. We choose instead to call the set of NEF with $V_{F}(\mathbf{m})$ as in (3.1) the Casalis class of NEF; it generalizes the Morris class from $\mathbb{R}$ to $\mathbb{R}^{n}$. Casalis has made an exhaustive study and shows that there are exactly $(2 n+4)$ such types. The following definition makes precise the term "type."

Definition 3.1. Let $\mu \in \mathscr{M}\left(\mathbb{R}^{n}\right)$ and let $\Lambda(\mu)$ (called the Jørgensen set) be the set of positive numbers $\lambda$ such that there exists a measure $\mu_{\lambda} \in \mathscr{M}\left(\mathbb{R}^{n}\right)$ for which $L_{\mu_{\lambda}}(\boldsymbol{\theta})=\left(L_{\mu}(\boldsymbol{\theta})\right)^{\lambda}$ is the Laplace transform of $\mu_{\lambda}$. Two NEFs $F_{1}$ and $F_{2}$ are said to be of the same type if there exist $\mu \in \mathscr{M}\left(\mathbb{R}^{n}\right), \lambda \in \Lambda(\mu)$ and an affinity $\phi$ in $\mathbb{R}^{n}$ such that with $\mu^{\prime}=\phi\left(\mu_{\lambda}\right)$ one has $F_{1}=F(\mu)$ and $F_{2}=F\left(\mu^{\prime}\right)$.

Below we give a list of the $(2 n+4)$ types together with their cumulant transforms and variance functions as given by Casalis. We also include the value of $\operatorname{det} k^{\prime \prime}(\boldsymbol{\theta})$ in each case, the computation of which is straightforward.

1. Poisson-Gaussian types $(n+1):(P G)_{k}, k=0, \ldots, n$,

$$
\mu(d \mathbf{X})=\left\{\sum_{j \in \mathbb{N}^{k}} \frac{\delta_{j}\left(d \mathbf{X}_{1}\right)}{j !}\right\} \frac{\exp \left\{-\frac{1}{2} \sum_{i=k+1}^{n} x_{i}^{2}\right\}}{(2 \pi)^{(n-k) / 2}}\left(d \mathbf{X}_{2}\right),
$$

where $\quad d X_{1}=d x_{1}, \ldots, d x_{k}, \quad d X_{2}=d x_{k+1}, \ldots, d x_{n}, \quad \Theta(\mu)=M_{F}=\mathbb{R}^{n}$, $k_{\mu}(\boldsymbol{\theta})=\sum_{i=1}^{k} e^{\theta_{i}}+\sum_{i=k+1}^{n}\left(\theta_{i}^{2} / 2\right), \operatorname{det} k_{\mu}^{\prime \prime}(\boldsymbol{\theta})=\exp \left(\theta_{1}+\cdots+\theta_{n}\right), \delta_{j}$ is Dirac mass at $j, \quad \mathbf{X}_{1}=\left(x_{1}, \ldots, x_{k}\right), \mathbf{X}_{2}=\left(x_{k+1}, \ldots, x_{n}\right)$ and $V_{F}(\mathbf{m})=$ $\operatorname{diag}\left(m_{1}, \ldots, m_{k}, 1, \ldots, 1\right)$.

2. Multinomial type $(M)$. Let $N$ be a positive integer, let $e_{0}$ be the null vector and let $\left(e_{1}, \ldots, e_{n}\right)$ be the canonical basis of $\mathbb{R}^{n}$. Define

$$
\mu=\left(\sum_{i=0}^{n} \delta_{e_{i}} \mathbb{R}\right)^{*^{N}}
$$

Then $\Theta(\mu)=\mathbb{R}^{n}, M_{F}=\left\{\mathbf{m} \in \mathbb{R}^{n} ; m_{i}>0, \forall i, \sum_{i=1}^{n} m_{i}<N\right\}, \quad k_{\mu}(\boldsymbol{\theta})=$ $N \log \left(1+\sum_{i=1}^{n} e^{\theta_{i}}\right)$,

$$
\operatorname{det} k_{\mu}^{\prime \prime}(\boldsymbol{\theta})=\exp \left\{-\frac{(n+1)}{N} k_{\mu}(\boldsymbol{\theta})+\theta_{1}+\cdots+\theta_{n}+n \log N\right\}
$$

and $V_{F}(\mathbf{m})=\operatorname{diag}\left(m_{1}, \ldots, m_{n}\right)-(\mathbf{m} \otimes \mathbf{m}) / N$.

3. Hyperbolic $(H)$. For $\lambda>0$ we define

$$
\mu_{\lambda}(d \mathbf{X})=\nu_{\lambda}\left(d X_{1}\right) \alpha_{\lambda+\sum_{i=1}^{n-1} x_{i}}(d y),
$$

where

$$
\nu_{\lambda}=\left(\delta_{0}-\sum_{i=1}^{n-1} \delta_{e_{i}}\right)^{*(-\lambda)}, \quad \alpha_{p}(d y)=\frac{2^{p-2}|\Gamma((p+i y) / 2)|^{2}}{\Gamma(p)(\Gamma(p / 2))^{2}} d y
$$


$p>0$ and $d \mathbf{X}_{1}=d x_{1}, \ldots, d x_{n-1}$. Then $\Theta(\mu)=\left\{\left(\theta_{1}, \ldots, \theta_{n}\right) ;-\pi / 2<\theta_{n}<\right.$ $\pi / 2$ and $\left.e^{\theta_{1}}+\cdots+e^{\theta_{n-1}}<\cos \theta_{n}\right\}, \quad M_{F}=(0, \infty)^{n-1} \times \mathbb{R}, \quad k_{\mu}(\boldsymbol{\theta})=$ $-p\left\{\log \left\{\cos \theta_{n}-\sum_{i=1}^{n-1} e^{\theta_{i}}\right\}\right.$,

$$
\operatorname{det} k_{\mu}^{\prime \prime}(\boldsymbol{\theta})=\exp \left\{\frac{(n+1)}{\lambda} k_{\mu}(\boldsymbol{\theta})+\theta_{1}+\cdots+\theta_{n}+n \log \lambda\right\}
$$

and

$$
V_{F}(\mathbf{m})=\operatorname{diag}\left(m_{1}, \ldots, m_{n-1}, \lambda+\sum_{i=1}^{n-1} m_{i}\right)+\frac{\mathbf{m} \otimes \mathbf{m}}{\lambda} .
$$

The NEF corresponding to the law of $\left(\mathbf{X}_{1}, \mathbf{Y}\right)$ is $F\left(\mu_{\lambda}\right)$, where $\mathbf{X}_{1}=$ $\left(X_{1}, \ldots, X_{n-1}\right)$ follows a negative multinomial law with parameter $\lambda$ and conditionally on $\mathbf{X}_{1}$ the random variable $Y$ follows the hyperbolic secant law with parameter $\left(\lambda+\sum_{i=1}^{n-1} X_{i}\right)$.

4. Negative multinomial-gamma types $(n+1) .(N M-G a)_{k}: k=0,1, \ldots, n$. For $0 \leq k \leq n$ and $\lambda>0$ we define $\mu_{\lambda}$ by

$$
\mu_{\lambda}(d \mathbf{X})=\nu_{\lambda}\left(d \mathbf{X}_{1}\right) \eta_{\left(\lambda+\sum_{i=1}^{k} x_{i}\right)}(d y) \prod_{i=k+2}^{n} \alpha_{y}\left(d z_{i}\right),
$$

where $\nu_{\lambda}$ is the measure defined in (3), $\eta_{p}(d y)=y^{p-1} /(\Gamma(p)) I_{\mathbb{R}}+(y) d y$ and $\alpha_{y}(d z)=1 / \sqrt{2 \pi y} \exp \left(-z^{2} / 2 y\right) d z$.

The NEF corresponding to the law of $(X, Y, Z)$ is $F\left(\mu_{\lambda}\right)$, where $X=$ $\left(X_{1}, \ldots, X_{k}\right)$ has the negative multinomial law with parameter $\lambda, Y$ conditionally on $X$ has a gamma law with shape parameter $\left(\lambda+\sum^{k} X_{i}\right)$ and $Z=\left(Z_{k+2}, \ldots, Z_{n}\right)$ conditionally on $(X, Y)$ is multinormal with covariance $Y I_{n-k-1}$. Note that this definition includes an arbitrary parameter in $\mathbb{R}^{k}$ for $X$, an arbitrary scale parameter for $Y$ and an arbitrary mean parameter for $Z$. The limiting cases $k=0, k=n-1$ and $k=n$ have obvious interpretations when an empty sum is replaced by zero. We then have

$$
\begin{aligned}
\Theta(\mu) & =\left\{\boldsymbol{\theta} \mid-e^{\theta_{1}}-\cdots-e^{\theta_{k}}-\theta_{k+1}-\frac{\theta_{k+2}^{2}}{2} \cdots-\frac{\theta_{n}^{2}}{2}>0\right\}, \\
M_{F} & =(0, \infty)^{k+1} \times \mathbb{R}^{n-k-1}, \\
k_{\mu}(\boldsymbol{\theta}) & =-\lambda \log \left[-\sum_{i=1}^{k} e^{\theta_{i}}-\theta_{k+1}-\sum_{i=k+2}^{n} \frac{\theta_{i}^{2}}{2}\right], \\
\operatorname{det} k_{\mu}^{\prime \prime}(\boldsymbol{\theta}) & =\exp \left\{\frac{n+1}{\lambda} k_{\mu}(\boldsymbol{\theta})+\theta_{1}+\cdots+\theta_{n}+n \log \lambda\right\}
\end{aligned}
$$

and

$$
V_{F}(\mathbf{m})=\operatorname{diag}\left(m_{1}, \ldots, m_{k}, 0, m_{k+1}, \ldots, m_{k+1}\right)+\frac{\mathbf{m} \otimes \mathbf{m}}{\lambda} .
$$

We now prove the following theorem. 
Theorem 3.1. Let $\mu \in \mathscr{M}\left(\mathbb{R}^{n}\right)$ such that $F=F(\mu)$ belongs to the Casalis class [thus $V_{F}(\mathbf{m})$ is given by (3.1)]. Let $\nu_{0}$ be defined from $\mu$ as in Theorem 2.2. Then $F\left(\nu_{0}\right)$ and $F(\mu)$ are of the same type.

Proof. From the above listing we see by inspection that there exists $(\mathbf{A}, b, c) \in \mathbb{R}^{n} \times \mathbb{R} \times \mathbb{R}$ such that

$$
\operatorname{det} k_{\mu}^{\prime \prime}(\boldsymbol{\theta})=\exp \left\{b k_{\mu}(\boldsymbol{\theta})+\langle\boldsymbol{\theta}, \mathbf{A}\rangle+c\right\} .
$$

From Theorem 2.2 we see that

$$
\begin{aligned}
k_{\nu_{0}}(\boldsymbol{\theta}) & =(n+1) k_{\mu}(\boldsymbol{\theta})+\log \left[\operatorname{det}\left\{V_{F(\mu)}\left(k_{\mu}^{\prime}(\boldsymbol{\theta})\right)\right\}\right] \\
& =(n+1+b) k_{\mu}(\boldsymbol{\theta})+\langle\boldsymbol{\theta}, \mathbf{A}\rangle+c .
\end{aligned}
$$

Hence

$$
k_{\nu_{0}}^{\prime}(\boldsymbol{\theta})=(n+1+b) k_{\mu}^{\prime}(\boldsymbol{\theta})+\mathbf{A} .
$$

Writing $\mathbf{m}^{*}=k_{\nu_{0}}^{\prime}(\boldsymbol{\theta})$ and $b^{*}=n+1+b$, we have

$$
\mathbf{m}^{*}=b^{*} \mathbf{m}+\mathbf{A} \text { and } V_{F\left(\nu_{0}\right)}\left(\mathbf{m}^{*}\right)=b^{*} V_{F(\mu)}\left(\frac{\mathbf{m}^{*}-\mathbf{A}}{b^{*}}\right) .
$$

This shows that $F\left(\nu_{0}\right)$ and $F(\mu)$ are of the same type.

This result generalizes Lindsay's verification for the Morris families for $n=1$.

The Casalis class does not cover the whole set of NEF with a quadratic variance. For instance, let $E$ be the space of $(d \times d)$ real symmetric matrices and let $S$ be the cone in $E$ of positive definite matrices. Consider the standard Wishart distribution $W(2 p, \Sigma)$ concentrated on $\bar{S}$ with $\Sigma$ in $S$ and $p$ in

$$
\Lambda=\left\{\frac{1}{2}, 1, \frac{3}{2}, \ldots, \frac{d-1}{2}\right\} \cup\left(\frac{d-1}{2},+\infty\right) .
$$

Then it is known that the NEF

$$
F_{p}=\{W(2 p, \Sigma) ; \Sigma \in S\}
$$

has a quadratic variance [Letac (1989)].

We now prove the following theorem.

Theorem 3.2. Let $\mu \in \mathscr{M}(E)$ such that $F(\mu)=F_{p}$ with $F_{p}$ given by (3.3) and $p$ in $\Lambda$ given by (3.2). Let $\nu_{0}$ be defined from $\mu$ as in Theorem 2.2. Then $F\left(\nu_{0}\right)=F_{p^{\prime}}$, where $p^{\prime}=p\{1+(d(d+1) / 2)\}+(d+1)$.

Proof. Without loss of generality we take $\mu=\mu_{p}$, where

$$
\mu_{p}(d X)=\frac{1}{\Gamma_{d}(p)}(\operatorname{det} X)^{p-1-((d-1) / 2)} I_{S}(X) d X,
$$


where

$$
\Gamma_{d}(p)=2^{d p} \pi^{(d(d-1)) / 4} \prod_{i=0}^{d-1} \Gamma\left(p-\frac{i}{2}\right), \quad \Theta\left(\mu_{p}\right)=-S,
$$

$S$ being the cone of $(d \times d)$ symmetric positive definite matrices and $p>$ $(d-1) / 2$. Furthermore,

$$
L_{\mu_{p}}(\theta)=\int_{S} \exp \left\{\frac{1}{2} \operatorname{tr}(\theta X)\right\} \mu_{p}(d X)=(\operatorname{det}(-2 \theta))^{-p}
$$

so that

$$
k_{\mu_{p}}(\theta)=-p \log (\operatorname{det}(-2 \theta))
$$

Then

$$
\operatorname{det} k_{\mu_{p}}^{\prime \prime}(\theta)=p^{(d(d+1)) / 2}(\operatorname{det}(-\theta))^{-d-1} .
$$

To see the above step we use three classical facts:

(a) The differential of $S \rightarrow \mathbb{R}, \theta \mapsto-\log (\operatorname{det} \theta)$ is $\theta^{-1}$.

(b) The differential of $S \rightarrow S, \theta \mapsto \theta^{-1}$ is $d \theta^{-1}=-\theta^{-1} d \theta \theta^{-1}$.

(c) If $A$ is any $(d \times d)$ matrix, the determinant of the linear endomorphism $\phi_{A}$ of the space of symmetric $(d \times d)$ matrices defined by $\phi_{A}(M)=$ $A M A^{t}$ is $\operatorname{det} \phi_{A}=(\operatorname{det} A)^{d+1}$.

Hence with $n=(d(d+1)) / 2$ (dimension of $E)$ we have

$$
k_{\nu_{0}}(\theta)=\frac{p^{\prime}}{p} k_{\mu_{p}}(\theta)+\log \text { const },
$$

where $p^{\prime}$ is defined in Theorem 3.2. Thus $F\left(\nu_{0}\right)=F_{p^{\prime}}$.

3.2. Rao-Blackwell estimation of $\operatorname{det} k_{\mu}^{\prime \prime}(\boldsymbol{\theta})$. The following theorem provides the key to obtaining the Rao-Blackwell estimator of the generalized variance of a NEF generated by $\mu \in \mathscr{M}\left(\mathbb{R}^{n}\right)$ in the case of $(n+1)$ observations.

THEOREM 3.3. Let $\mu \in \mathscr{M}\left(\mathbb{R}^{n}\right)$ and let $\nu_{0}$ be the image measure defined in Theorem 2.2. Denote by $\mu^{*(n+1)}$ the image measure of $\mu\left(d \mathbf{X}_{0}\right) \mu\left(d \mathbf{X}_{1}\right) \cdots$ $\mu\left(d \mathbf{X}_{n}\right)$ by the map $S_{0}$ of Theorem 2.2. Suppose that there exists $C(\mathbf{X})$ such that

$$
\nu_{0}(d \mathbf{X})=C(\mathbf{X}) \mu^{*(n+1)}(d \mathbf{X}) .
$$

Then $C\left(\mathbf{X}_{0}+\mathbf{X}_{1}+\cdots+\mathbf{X}_{n}\right)$ is the Rao-Blackwell estimator of $\operatorname{det} k_{\mu}^{\prime \prime}(\boldsymbol{\theta})$ based on $(n+1)$ observations $\mathbf{X}_{0}, \ldots, \mathbf{X}_{n}$. 
Proof. From Theorem 2.2 we have, for each $\boldsymbol{\theta} \in \Theta(\mu)$,

$$
\begin{aligned}
\operatorname{det} k_{\mu}^{\prime \prime}(\boldsymbol{\theta}) & =\frac{L_{\nu_{0}}(\boldsymbol{\theta})}{L_{\mu^{*(n+1)}}(\boldsymbol{\theta})} \\
& =\int_{\left(\mathbb{R}^{n}\right)^{n+1}} C\left(\mathbf{X}_{0}+\cdots+\mathbf{X}_{n}\right) \prod_{i=0}^{n} P(\boldsymbol{\theta}, \mu)\left(d \mathbf{X}_{i}\right) .
\end{aligned}
$$

As an illustration of Theorem 3.3 we give below the Rao-Blackwell estimators of the Casalis class as well as the Wishart families.

ExAmple 3.1. Cases of the Casalis class.

Case 1. Poisson-Gaussian types $(P G)_{k}, k=0,1, \ldots, n$, give for $x_{1} \neq 0$, $\ldots, x_{k} \neq 0$,

$$
C(\mathbf{X})=\frac{\nu_{0}(d \mathbf{X})}{\mu^{*(n+1)}(d \mathbf{X})}=\frac{\mu_{n+1} * \delta_{\mathbf{e}_{1}+\cdots+\mathbf{e}_{k}}(d \mathbf{X})}{\mu_{n+1}(d \mathbf{X})}=x_{1} \cdots x_{k} .
$$

Note that it is independent of the Gaussian component.

Case 2. In the multinomial case we have

$$
\begin{aligned}
L_{\nu_{0}}(\boldsymbol{\theta}) & =N^{n}\left(1+\sum_{i=1}^{n} \exp \left(\theta_{i}\right)\right)^{(n+1)(N-1)} \exp \left(\sum_{i=1}^{n} \theta_{i}\right), \\
L_{\mu^{*}(n+1)}(\boldsymbol{\theta}) & =\left(1+\sum_{i=1}^{n} \exp \left(\theta_{i}\right)\right)^{(n+1) N} .
\end{aligned}
$$

Note that $\nu_{0}$ is concentrated on the tetrahedron $T_{0}$ in $\mathbb{R}^{n}$ with vertices $\mathbf{p}=\sum_{i=1}^{n} \mathbf{e}_{i},(n+1)(N-1) \mathbf{e}_{j}+\mathbf{p}, j=1,2, \ldots, n$, while $\mu^{*(n+1)}$ is concentrated on the tetrahedron $T_{1}$ in $\mathbb{R}^{n}$ with vertices $\mathbf{0},(n+1) N \mathbf{e}_{j}$, which contain $T_{0}$. If $\mathbf{X}=\left(x_{1}, \ldots, x_{n}\right)$ is in $T_{0}$, then

$$
\nu_{0}(\mathbf{X})=N^{n}[(n+1)(N-1)] ! /\left(x_{1}-1\right) ! \cdots\left(x_{n}-1\right) !,
$$

whereas $\mu^{*(n+1)}(\mathbf{X})=[(n+1) N] ! / x_{1} ! \cdots x_{n}$ !

Thus

$$
\begin{aligned}
C(\mathbf{X}) & =\frac{\nu_{0}(\mathbf{X})}{\mu^{*(n+1)}}(\mathbf{X}) \\
& =\frac{N^{n}[(n+1)(N-1)]}{[(n+1) N]} x_{1} \cdots x_{n} \quad \text { if } X \in T_{0}
\end{aligned}
$$

and $C(\mathbf{X})=0$ if $X \notin T_{0}$.

Case 3. For the hyperbolic type $(H)$ we have

$$
L_{\mu_{\lambda}^{*(n+1)}}(\boldsymbol{\theta})=\left(\cos \theta_{n}-\sum_{i=1}^{n-1} e^{\theta_{i}}\right)^{-\lambda(n+1)}=L_{\mu_{\lambda(n+1)}}(\boldsymbol{\theta}),
$$


while from Theorem 2.2,

$$
L_{\nu_{0}}(\boldsymbol{\theta})=\lambda^{n} L_{\mu_{(\lambda+1)(n+1)}}(\boldsymbol{\theta}) \exp \left(\sum_{i=1}^{n-1} \theta_{i}\right) .
$$

Hence for $x_{1} \neq 0, \ldots, x_{k} \neq 0$,

$$
C(\mathbf{X})=\frac{\nu_{0}(d \mathbf{X})}{\mu_{\lambda(n+1)}(d \mathbf{X})}=\frac{\lambda^{n} \mu_{(\lambda+1)(n+1)} * \delta_{\mathbf{e}_{1}+\cdots+\mathbf{e}_{n-1}}(d \mathbf{X})}{\mu_{\lambda(n+1)}(d \mathbf{X})} .
$$

[Observe that the density corresponding to $\nu_{0}$ suffers a translation for the support of $\left(x_{1}, \ldots, x_{k}\right)$ by one unit because of the factor $\exp \left(\sum_{i=1}^{n-1} \theta_{i}\right)$.]

Letting $\beta=\sum_{j=1}^{n-1} x_{j}+\lambda_{1}$, we obtain

$$
C(\mathbf{X})=\frac{2^{4} \lambda^{n}\left(\beta^{2}+x_{n}^{2}\right) x_{1} \cdots x_{n-1}}{\lambda_{1}\left(\lambda_{1}+1\right) \cdots\left(\lambda_{1}+n\right) \beta^{2}} .
$$

Case 4. For the negative multinomial-gamma type, $(N M-G a)_{k}, k=$ $0,1, \ldots, n$, we have for $x_{1} \neq 0, \ldots, x_{k} \neq 0$,

$$
L_{\mu}(\boldsymbol{\theta})=\left[-\sum_{i=1}^{k} e^{\theta_{i}}-\theta_{k+1}-\sum_{i=k+2}^{n} \frac{\theta_{i}^{2}}{2}\right]^{-\lambda}
$$

so that $L_{\mu^{*}(n+1)}(\boldsymbol{\theta})=\left[L_{\mu}(\boldsymbol{\theta})\right]^{n+1}$, while from Theorem 2.2,

$$
L_{\nu_{0}}(\boldsymbol{\theta})=\lambda^{n}\left[-\sum_{i=1}^{k} \exp \left(\theta_{i}\right)-\theta_{k+1}-\sum_{i=k+2}^{n} \frac{\theta_{i}^{2}}{2}\right]^{-(\lambda+1)(n+1)} \exp \left(\sum_{i=1}^{k} \theta_{i}\right) .
$$

Thus we obtain

$$
C(\mathbf{X})=\frac{\lambda^{n} \Gamma(\lambda(n+1))}{\Gamma((\lambda+1)(n+1))} x_{1} \cdots x_{k}\left(x_{k+1}\right)^{n-k+1} .
$$

The Rao-Blackwell estimator of $\operatorname{det} k_{\mu}^{\prime \prime}(\boldsymbol{\theta})$ is therefore $C\left(\mathbf{X}_{0}+\cdots+\mathbf{X}_{n}\right)$ with $C(\mathbf{X})$ as given above.

ExAMPLE 3.2. For the Wishart families we observe that (Theorem 3.2)

$$
\nu_{0}(d X)=\frac{2^{d(d+1)} p^{(d(d+1)) / 2}}{\Gamma_{d}\left(p_{0}\right)}(\operatorname{det} X)^{p_{0}-1-((d-1) / 2)} I_{S}(X)(d X)
$$

with $p_{0}=p\left[1+\frac{1}{2}(d(d+1))\right]+(d+1)$, while

$$
\mu^{*(n+1)}(d X)=\frac{1}{\Gamma_{d}\left(p_{0}-d-1\right)}(\operatorname{det} X)^{p_{0}-(d+1)-1-((d-1) / 2)} I_{S}(X)(d X)
$$

so that

$$
C(X)=\frac{2^{d(d+1)} p^{(d(d+1)) / 2} \Gamma_{d}\left(p_{0}-d-1\right)}{\Gamma_{d}\left(p_{0}\right)}(\operatorname{det} X)^{d+1} .
$$


If $X_{1}, \ldots, X_{m}$ are i.i.d. random variables with Wishart distribution $W(2 p, \Sigma)$ with $\theta=-\Sigma^{-1} / 2$, then from the well-known formula [Muirhead (1982)]

$$
\mathbb{E}\left((\operatorname{det} X)^{r}\right)=(\operatorname{det}(-2 \theta))^{r} 2^{d r} \frac{\Gamma_{d}(r+p)}{\Gamma_{d}(p)},
$$

we have

$$
\mathbb{E}\left(\operatorname{det}\left(X_{1}+\cdots+X_{m}\right)\right)^{r}=(\operatorname{det}(-2 \theta))^{r} 2^{d r} \frac{\Gamma_{d}(r+m p)}{\Gamma_{d}(p)} .
$$

Since $\operatorname{det} k_{\mu_{p}}^{\prime \prime}(\theta)=\operatorname{const}(\operatorname{det}(-2 \theta))^{d+1}$ for $r=d+1$, we obtain

$$
\mathbb{E}\left(\operatorname{det}\left(X_{1}+\cdots+X_{q}\right)\right)^{d+1}=\left(\operatorname{det} k_{\mu_{p}}^{\prime \prime}\right) \times \text { const. }
$$

Thus with $q$ observations $X_{1}, \ldots, X_{q}, \operatorname{const}\left(\operatorname{det}\left(X_{1}+\cdots+X_{q}\right)\right)^{d+1}$ is the Rao-Blackwell estimator of $\operatorname{det} k_{\mu_{p}}^{\prime \prime}(\theta)$. Hence our method is not very efficient since it gives the estimator only for the case $q=1+(d(d+1) / 2)$.

Our results in the case of NEF with simple quadratic variance structure can be regarded as a partial (since we consider $n+1$ observations) generalization of the result for Morris families in one dimension, namely, the Rao-Blackwell estimator of $k_{\mu}^{\prime \prime}(\theta)$ is $V_{F(\mu)}\left(\left(X_{1}+\cdots+X_{q}\right) / q\right) q /(q+a)$, where $V_{F(\mu)}(m)=a m^{2}+b m+c[\operatorname{Letac}(1992)]$.

3.3. Characterization of Gaussian laws in $\mathbb{R}^{n}$. Theorem 2.2 also implies the following remarkable fact about Gaussian laws in $\mathbb{R}^{n}$. If $\mathbf{X}_{0}, \mathbf{X}_{1}, \ldots, \mathbf{X}_{n} \in$ $\mathbb{R}^{n}$, then

$$
v\left(\mathbf{X}_{0}, \mathbf{X}_{1}, \ldots, \mathbf{X}_{n}\right)=\frac{1}{(n+1) !} \operatorname{det}\left[\begin{array}{cccc}
1 & 1 & \cdots & 1 \\
\mathbf{X}_{0} & \mathbf{X}_{1} & \cdots & \mathbf{X}_{n}
\end{array}\right]
$$

is the algebraic volume of the tetrahedron with vertices $\mathbf{X}_{0}, \ldots, \mathbf{X}_{n}$. Let $\mu$ in $\mathscr{M}\left(\mathbb{R}^{n}\right)$ be also a probability; consider the two probabilities in $\left(\mathbb{R}^{n}\right)^{n+1}$ :

$$
\begin{aligned}
& P_{1}=\mu \otimes \cdots \otimes \mu, \\
& P_{0}=K v^{2} P_{1},
\end{aligned}
$$

where $K$ is a normalization constant. Denote by $Q_{0}$ and $Q_{1}$ the respective images of $P_{0}$ and $P_{1}$ by the map

$$
\left(\mathbf{X}_{0}, \mathbf{X}_{1}, \ldots, \mathbf{X}_{n}\right) \mapsto \mathbf{X}_{0}+\cdots+\mathbf{X}_{n}
$$

A reformulation of Theorem 2.2 gives

$$
L_{Q_{0}}(\boldsymbol{\theta})=L_{Q_{1}}(\boldsymbol{\theta})\left(\operatorname{det}\left(k_{\mu}^{\prime \prime}(\boldsymbol{\theta})\right) / \operatorname{det}\left(k_{\mu}^{\prime}(0)\right)\right) .
$$

Now suppose that $\mu$ is Gaussian (with any mean and covariance). Then $Q_{0}$ and $Q_{1}$ have the same distribution (obviously a Gaussian one). It is tantaliz- 
ing to think of a converse. For $n=1$ it is trivial. For general $n$, it relies on the following delicate result of Pogorelov (1978).

THEOREM 3.4. Let $f$ be a $C^{\infty}$ convex function on $\mathbb{R}^{n}$ such that the determinant of the Hessian matrix $f^{\prime \prime}$ is a constant. Then $f^{\prime \prime}$ itself is a constant.

This is a reformulation of Pogorelov's result (on page 90). We then have the following characterization of Gaussian laws in $\mathbb{R}^{n}$ :

THEOREM 3.5. Let $\mu, Q_{0}$ and $Q_{1}$ be as defined above. Assume further that the Laplace transform of $\mu$ is finite everywhere in $\mathbb{R}^{n}$. Then $Q_{0}=Q_{1}$ if and only if $\mu$ is Gaussian.

Proof. The "if" part is obvious from (3.4). The "only if" part comes also from (3.4), which gives that $\boldsymbol{\theta} \mapsto \operatorname{det} k_{\mu}^{\prime \prime}(\boldsymbol{\theta})$ is a constant. Since $\boldsymbol{\theta} \mapsto k_{\mu}(\boldsymbol{\theta})$ is a real analytic strictly convex function which is defined on all $\mathbb{R}^{n}$, Pogorelov's theorem applies and $k_{\mu}^{\prime \prime}(\boldsymbol{\theta})$ is a constant. This implies that $\mu$ is Gaussian with covariance $k_{\mu}^{\prime}(\boldsymbol{\theta})$ and arbitrary mean.

Acknowledgments. We are very grateful to Gérard Letac and Muriel Casalis for their constructive comments during the preparation of this paper. Our special gratitude to M. Casalis for showing us a preprint of her work. We sincerely thank the Associate Editor and the two referees, who provided extensive comments which helped remove many inaccuracies and ambiguities. One referee was kind enough to supply the reference to Pogorelov's result, which we were unaware of.

\section{REFERENCES}

Casalis, M. (1992). Les familles exponentielles sur $\mathbb{R}^{2}$ de fonction-variance $V(m)=a m \otimes m+$ B(m) + C. C. R. Acad. Sci. Paris Sér. I Math. 314 635-638.

CAsalis, M. (1994). Les $2 n+4$ types de familles exponentielles naturelles sur $\mathbb{R}^{n}$ à fonction variance quadratique simple. C. R. Acad. Sci. Paris Sér. I Math. 318 261-264.

KoKonendJI, C. C. (1993). Familles exponentielles naturelles réelles de fonction variance en $R \Delta+Q \sqrt{\Delta}$. Thèse, Report 1467, Univ. Paul Sabatier, Toulouse.

KoKonendJi, C. C. and SEShADRI, V. (1992). La méthode de Lindsay appliquée à la construction de familles exponentielles de fonction variance de degré 4 en $\sqrt{m}$. C. R. Acad. Sci. Paris Sér. I Math. 314 305-308.

Kokonendj, C. C. and SeshadRi, V. (1994). Le déterminant de la dérivée seconde d'une transformée de Laplace est une transformée de Laplace. C. R. Acad. Sci. Paris Sér. I Math. 318 361-366.

LETAC, G. (1989). A characterization of the Wishart exponential families by an invariance property. J. Theoret. Probab. 2 71-86.

LETAC, G. (1992). Lectures on natural exponential families and their variance functions. IMPA, Rio de Janeiro.

Lindsay, B. G. (1989). On the determinant of moment matrices. Ann. Statist. 17 711-721.

MorRIs, C. N. (1982). Natural exponential families with quadratic variance functions. Ann. Statist. 10 65-80. 
Muirhead, R. J. (1982). Aspects of Multivariate Statistical Theory. Wiley, New York. Pogorelov, A. V. (1978). The Minkowski Multidimensional Problem. Wiley, New York.

Pólya, G. and Szegö, G. (1972). Problems and Theorems in Analysis 1. Springer, Berlin.

WiLKs, S. S. (1932). Certain generalizations in the analysis of variance. Biometrika 24 471-494.

Université de Pau et des Pays de l'Adour Laboratoire de Mathématiques APPliquées CNRS URA 1204

AVEnUe DE L'UNIVERSITÉ $64000 \mathrm{PAU}$

FRANCE
Department of Mathematics and Statistics MCGILl UNIVERSITY

805 Sherbrooke Street West

MONTREAL, QUEBEC

Canada H3A 2K6 